\section{CHALLENGES IN DIAGNOSIS AND STAGING OF LUNG CANCER}

Jeffrey L. Myers

Department of Pathology, Michigan Medicine, Ann Arbor, MI, USA

Pathology plays an important role in diagnosis and management of patients with non-small cell lung carcinoma (NSCLC). The current (2015) WHO classification of lung tumours includes histologic and immunophenotypic (IHC) criteria. Adenocarcinomas are the primary focus of shifting nomenclature, emphasising histologic patterns with clinical and biological relevance. Minimally invasive and lepidic-predominant non-mucinous adenocarcinomas represent well-differentiated tumours, while solid and/or micropapillary growth patterns signify poorly-differentiated adenocarcinomas. Poorly-differentiated carcinomas that cannot be classified on the basis of histology alone can instead be subclassified using a limited IHC panel targeting TTF-1 and p63/ p40. For adenocarcinomas with non-invasive (lepidic) components, pT staging hinges on the size and extent of only the invasive component. Multifocal tumours are staged based on a combination of gross growth characteristics and histologic findings. Pathology's role extends beyond diagnosis and staging in response to expectations that cells and tissues will be available for expanded biomarker testing in patients with advanced stage disease. Testing strategies for predictive biomarkers frequently include a combination of immunohistochemistry and increasingly comprehensive molecular profiling as single gene assays become less common. Panels are constructed to balance the number of actionable targets over and against our ability to test extremely small amounts of viable tumour.

\section{DIAGNOSING MESOTHELIOMA AND OTHER DIFFUSE PLEURAL TUMOURS... YOU CAN DO THIS AT HOME!}

Jeffrey L. Myers

Department of Pathology, Michigan Medicine, Ann Arbor, MI, USA

Accurate diagnosis of pleural tumours is increasingly important as tailored therapeutic strategies continue to evolve. Biopsy diagnosis of diffuse mesotheliomas begins with recognition of characteristic cytologic and architectural features in classical cases. Immunohistochemistry (IHC) can be useful for establishing the diagnosis with greater confidence in unusual histologic variants, such as solid, pleomorphic and lymphohistiocytoid subtypes. Perhaps the greatest value of IHC is in separating epithelioid mesotheliomas from metastatic carcinomas. IHC can also be helpful in identifying epithelioid haemangioendotheliomas with diffuse pleural involvement, a rare epithelioid neoplasm that occasionally mimics the clinical, radiologic, and histologic features of mesothelioma. IHC is less helpful for separating mesotheliomas with sarcomatoid and mixed histology from sarcomatoid carcinomas and soft tissue sarcomas. Desmoplastic mesotheliomas are a distinctive variant that often poses diagnostic challenges especially in small biopsies. Recognition of the characteristic storiform 'patternless pattern' of Stout and an invasive growth pattern are often key to diagnosis. Separating benign mesothelial proliferations from epithelioid mesotheliomas can be more challenging. There are now powerful immunohistochemical and molecular tools to help with this distinction, including IHC for BAP-1 and MTAP. p16 (9p21) deletion remains an important adjunctive strategy when IHC fails to resolve the diagnosis.

\section{GETTING COMFORTABLE WITH UIP}

Jeffrey L. Myers

Department of Pathology, Michigan Medicine, Ann Arbor, MI, USA

Pathology continues to play a key role in accurate diagnosis and subclassification of diffuse fibrotic lung disease. Usual interstitial pneumonia (UIP) is the flagship, accounting for about two-thirds of patients with idiopathic interstitial pneumonia who undergo lung biopsy. The histopathologic hallmark and chief diagnostic criterion for UIP is a low magnification appearance of patchy dense fibrosis, architectural distortion including honeycomb change, and qualitative and temporal variability resulting in a characteristic variegated appearance. Temporal variability includes fibroblast foci which are small foci of abnormal wound healing representing acute lung injury in a background of scarred lung. In its classic form this pattern of fibrosis is the single most important predictor of disease behaviour at the time of diagnosis. Fibrotic non-specific interstitial pneumonia (NSIP) differs in being qualitatively uniform, diffuse in distribution, and without significant architectural distortion. These criteria have emerged over decades of retrospective studies focusing on surgical lung biopsies. Transbronchial cryobiopsies compete with surgical lung biopsies as measured by their diagnostic yield and may play an increasingly important role in managing these patients. Multidisciplinary discussion is an emerging diagnostic standard for which biopsy interpretation remains a foundational element.

\section{DIFFUSE LUNG DISEASES WORTH KNOWING ABOUT}

\section{Jeffrey L. Myers}

Department of Pathology, Michigan Medicine, Ann Arbor, MI, USA

Smoking-related interstitial fibrosis (SRIF) and Langerhans cell histiocytosis $(\mathrm{LCH})$ are fibrotic forms of smoking-related lung disease that are sometimes confused with UIP in lung biopsies. SRIF is a common incidental finding typically seen in lobectomies for lung cancer in patients with a smoking history. It can also occur in diagnostic lung biopsies from patients with other types of diffuse lung diseases, including usual interstitial pneumonia (UIP). In either of these circumstances, SRIF remains an incidental finding of limited significance beyond corroborating a smoking history. In a subset of patients, SRIF may account for a form of mild restrictive lung disease. Pulmonary LCH is a lesion confined to the lungs of cigarette smokers, some of whom may be asymptomatic at the time of diagnosis. The key to diagnosis is recognition of the distinctive bronchiolocentric distribution of stellate nodules with associated 'scar emphysema'. Lymphangioleiomyomatosis (LAM) occurs almost exclusively in women who tend to have characteristic radiologic findings that may overlap with those seen in LCH. Histologic diagnosis hinges on a distinct constellation of abnormalities including a population of smooth muscle-like cells with cytologic and immunophenotypic features indistinguishable from PEComa. 